\title{
Philosophiques
}

\section{Claude Lafleur, Quatre introductions à la philosophie au XIII siècle. Textes critiques et étude historique, Montréal, Institut d'études médiévales, et Paris, Vrin, 1988, XX + 428pages.}

\section{Claude Panaccio}

Volume 17, numéro 2, automne 1990

URI : https://id.erudit.org/iderudit/027126ar

DOI : https://doi.org/10.7202/027126ar

Aller au sommaire du numéro

Éditeur(s)

Société de philosophie du Québec

ISSN

0316-2923 (imprimé)

1492-1391 (numérique)

Découvrir la revue

Citer ce compte rendu

Panaccio, C. (1990). Compte rendu de [Claude Lafleur, Quatre introductions à la philosophie au XIII ${ }^{e}$ siècle. Textes critiques et étude historique, Montréal,

Institut d'études médiévales, et Paris, Vrin, 1988, XX + 428pages.]

Philosophiques, 17(2), 201-203. https://doi.org/10.7202/027126ar d'utilisation que vous pouvez consulter en ligne.

https://apropos.erudit.org/fr/usagers/politique-dutilisation/ 


\title{
COMPTES RENDUS
}

CLAUDE LAFLEUR, Quatre introductions d la pbilosopbie auXII siècle. Textes critiques et étude historique, Montréal, Institut d'études médiévales, et Paris, Vrin, 1988, XX + 428pages.

\author{
par Claude Panaccio
}

Il y avait dans l'université médiévale quelque chose que l'on appelait "philosophie .. À ceux qui en douteraient, les quatre textes édités ici par Claude Lafleur en témoignent de manière irréfutable. Ce sont des guides à l'intention des étudiants de la faculté des arts de l'Université de Paris. Chacun présente en abrégé une sorte de tableau raisonné de l'ensemble ou d'une partie des disciplines enseignées dans cette faculté qui assurait, comme on sait, pour les jeunes gens de quatorze à vingt ans environ, une longue propédeutique aux études plus avancées en théologie, en droit ou en médecine. Le mot " philosophie - est, dans chacun de ces traités, le terme-clé qui sert à chapeauter toutes les matières non théologiques. Trois des quatre manuels, d'ailleurs, consacrent d'entrée de jeu quelques lignes, voire quelques pages, à le définir. Plusieurs formules y sont proposées qui reviennent en définitive à dire que la philosophie est l'étude par des moyens purement naturels - c'est-à-dire par la raison surtout - de tout ce qui existe, de l'être humain dans son ensemble et jusque, selon certaines définitions, des choses divines elles-mêmes, dans la mesure du moins où elles sont accessibles à la seule réflexion sans l'aide de la foi révélée.

Les quatre traités datent des années 1230 à 1255 environ. Pris conjointement, ils manifestent avec évidence l'entrée en force, vers cette époque, de la science gréco-latine et de l'aristotélisme en particulier dans l'université médiévale, et la constitution sous le nom de * philosophie d'un savoir véritablement profane. Trois de ces «introductions " sont anonymes : les Accessus philosophorum, la Pbilosophica disciplina et le très court Compendium circa quadrivium. La quatrième, une Divisio scientiarum, est d'un certain Arnoul de Provence, qui atteignit apparemment comme maître de l'Université de Paris une renommée assez enviable, mais dont on ne connaît par ailleurs aucun autre écrit. Ce ne sont pas, du reste, des ouvrages susceptibles par leur profondeur d'immortaliser leurs auteurs, fussent-ils un jour bien identifiés. Leur intérêt, comme le signale Lafleur, tient à ce qu'ils révèlent de l'enseignement qui avait cours à la faculté des arts, de son contenu doctrinal aussi bien que de ses programmes officiels.

L'édition des textes proprement dite occupe, avec son apparat critique et un long cortège de notes explicatives, quelque deux cents pages dans le livre, qui en compte au total plus de quatre cents. Le reste est 
surtout consacré à une «Préface * en trois chapitres. L'auteur y procède d'abord à une fastidieuse description de tous les manuscrits qu'il a utilisés, la plus détaillée que j'aie jamais vue, je crois bien (7-75); puis, au chapitre 2 , à une classification des manuscrits pour chacun de ses quatre traités, ce qu'on appelle en langage de métier l'établissement de * stemmas * (77-122). Le lecteur normal, à vrai dire, s'il n'est pas un professionnel de l'édition de textes, peut se permettre d'aller directement à l'intéressant chapitre 3 : «Présentation des quatre opuscules » (123-154), où se trouvent entre autres choses un éclaicant "portrait sommaire de l'enseignement à la faculté des arts de Paris» (141-144) et, surtout, un bilan dense (trop court même, me semble-t-il, par rapport à la taille démesurée des chapitres précédents) de l'*apport des introductions à la philosophie à notre connaissance de l'enseignement de la faculté des arts de Paris» (146-154).

Viennent ensuite les traités eux-mêmes (en latin, comme de raison). Regardons, à titre d'exemple, celui d'Arnoul, le plus complet et le plus intéressant, à mon avis, tant par le style, assez recherché, que par le contenu. Après une introduction générale et un essai de définition de la philosophie, de la sagesse et de la science, il s'attaque à une taxinomie du savoir profane. La philosophie au sens large est d'abord divisée en sciences mécaniques et sciences libérales. Les premières, au nombre de sept (le tissage, la navigation, l'art militaire, l'agriculture, le théâtre, la médecine et la divination) sont expédiées en une courte section et le reste du traité est consacré à la philosophie dite libérale, qui, seule, intéresse directement les étudiants auxquels s'adresse le maître parisien. Elle se répartit en trois : la philosophie naturelle, la philosophie rationnelle (ou étude du discours) et la philosophie pratique. La première, à son tour, prise au sens large, comprend la métaphysique, les sciences mathématiques (c'est-à-dire le quadrivium classique : l'arithmétique, la musique, la géométrie et l'astronomie) et, finalement, la philosophie naturelle au sens étroit (c'est-à-dire l'étude des corps matériels mobiles). La philosophie dite pratique est identifiée à la morale, d'inspiration aristotélicienne surtout et comprenant, en plus de l'éthique individuelle, la politique et l'économie familiale. Quant à la philosophie rationnelle, elle correspond au bon vieux trivium la grammaire, la logique et la rhétorique.

Chacune de ces subdivisions est traitée en une cinquantaine de lignes, où la discipline considérée est d'abord définie, puis resubdivisée en ses diverses parties. L'ensemble fournit ainsi le tableau encyclopédique d'un savoir structuré qui ne dépend en rien de la foi religieuse ni de la révélation.

Les trois autres traitês procèdent à peu près de la même manière, mais sont, à des degrés divers, moins exhaustifs. Le Compendium circa quadrivium, notamment, découvert par Lafleur lui-même, se limite, comme l'indique le titre qu'il lui a donné (le manuscrit n'en portait aucun), aux quatre sciences mathématiques. Les Accessus philosophorum accordent aussi au quadrivium la place d'honneur, mais y ajoutent, il est intéressant de le noter, une section consacrée au De consolatione philosophiae de Boèce, une autre au Timée de Platon et une dernière, enfin, assez longue, à la rhétorique. Quant à la Philosopbica disciplina, elle suit à peu de choses près le même plan que la Divisio d'Arnoul, dont 
elle est, du reste, selon la démonstration de Lafleur, l'une des sources immédiates.

Tout cela éclaire beaucoup, au total, l'allure de l'enseignement à la faculté des arts vers le milieu du XIII ${ }^{e}$ siècle. On y voit s'organiser les connaissances et les disciplines, les démarcations se tracer, les spécialisations s'esquisser. Claude Lafleur a produit là, avec une compétence et une minutie peu communes, un travail fort utile qu'apprécieront au plus haut point tous les treizièmistes, d'où qu'ils soient.

Université du Québec à Trois-Rivières 